\title{
Holographic fermionic system with dipole coupling on Q-lattice
}

\author{
Yi Ling, ${ }^{a, b}$ Peng Liu, ${ }^{a}$ Chao Niu, ${ }^{a}$ Jian-Pin $\mathbf{W u}{ }^{c, b}$ and Zhuo-Yu Xian ${ }^{a}$ \\ ${ }^{a}$ Institute of High Energy Physics, Chinese Academy of Sciences, \\ Beijing 100049, China \\ ${ }^{b}$ State Key Laboratory of Theoretical Physics, \\ Institute of Theoretical Physics, Chinese Academy of Sciences, \\ Beijing 100190, China \\ ${ }^{c}$ Department of Physics, School of Mathematics and Physics, Bohai University, \\ Jinzhou 121013, China \\ E-mail: lingy@ihep.ac.cn, liup51@ihep.ac.cn, niuc@ihep.ac.cn, \\ jianpinwu@gmail.com, xianzy@ihep.ac.cn
}

ABSTRACT: We construct a holographic model for a fermionic system on Q-lattice and compute the spectral function in the presence of a dipole coupling. Both key features of doped Mott insulators, the dynamical generation of a gap and spectral weight transfer, are observed when adjusting the value of the coupling parameter $p$. Of particular interest is that when the background is in a deep insulating phase, the Mott gap opens much easier with a smaller coupling parameter in comparison with a metallic background. The effects of lattice parameters on the width of the gap $\Delta / \mu$ are studied and a turning point is observed near the critical regime of metal-insulator transitions of the background. Furthermore, the temperature dependence of the spectral function is studied. Finally, we also observe that the anisotropic Q-lattice generates anisotropic peaks with different magnitudes, indicating that insulating and metallic phases arise in different directions.

KeYwords: Holography and condensed matter physics (AdS/CMT), AdS-CFT Correspondence

ARXIV EPRINT: 1410.7323 


\section{Contents}

1 Introduction 1

2 Holographic Q-lattice geometry 2

3 Dirac equation 3

4 Mott transition on Q-lattice 5

4.1 Free fermionic spectral function 5

4.2 The Mott transition $\quad 6$

$\begin{array}{ll}4.3 \text { The dynamics at different temperatures } & 10\end{array}$

4.4 Fermi surface and gap on anisotropic Q-lattice 12

5 Conclusion and discussion $\quad 12$

\section{Introduction}

To understand and describe Mott metal-insulator transition (MIT) is a long-standing and widely known difficult problem in condensed matter physics because it involves a strongly correlated electron system, in which the conventional theoretical tools prove of little help and non-perturbative techniques are called for. At this stage, holography may provide insights into the associated mechanisms of these strongly correlated electron systems by building a gravitational dual model which is usually solvable in the large $N$ limit. Some excellent examples are the holographic superconductor [1-3] and holographic (non)-Fermi liquid [4-14].

As early as 1930s, it was reported that many transition-metal oxides (such as $\mathrm{NiO}$ ) with partially filled bands show insulating behavior. Peierls ascribed that to the strong electron-electron correlation. And then, Mott made a series of pioneering work towards understanding how electron-electron correlations could explain the insulating state [1519]. Roughly speaking, the main idea of Mott is that the transition from metal to insulator occurs as lattice constant increases. Subsequently, this idea of Mott is formalized in the Hubbard model in which the Mott transition depends on the competition between the kinetic energy $\left(E_{k} \sim t\right)$ and the on-site Coulomb repulsion $U$. The metallic behavior prevails if the kinetic energy $t$ overcomes the Coulomb energy $U$ while insulating phase is favored for $U / t \gg 1$ and a gap opens in the single-particle excitation spectrum, resulting in a Mott transition at a critical ratio of $U / t$.

By adding probe fermions with dipole coupling in RN-AdS black hole [20, 21], a Mott gap opens dynamically, which exhibits two key features of doped Mott insulator, i.e., the dynamical generation of a gap and spectral weight transfer. And then, the dipole coupling 
effects have also been studied in more general geometries in [22-29]. Along this line, here we shall study the holographic fermionic system with dipole coupling in Q-lattice geometry.

Holographic Q-lattice model is firstly proposed in [30], which is similar to the construction of Q-balls [31]. Some extensive studies have been presented in [32-35]. In this framework, the translational symmetry is broken and MIT is observed through the study of optical conductivity. Different from the holographic scalar lattice and ionic lattice constructed in [36-41], which involve solving the PDEs and need a hard numerical work, but here we only need solve ODEs for Q-lattice. In particular, MIT is not observed in scalar lattice or ionic lattice background yet. The difficulties of numerical calculation prevent one from dropping the temperature down to an extremely low level in this context. ${ }^{1}$ Here, we are interested in understanding if there is a relationship between the phase (metal/insulator) the background geometry with Q-lattice is in and that the fermionic excitations on Q-lattice are in. Therefore, in this paper we shall study the fermionic excitations by adding a probe fermion with dipole coupling in Q-lattice geometry.

This paper is organized as follows. In section 2, based on the holographic model originally presented in [30], which introduces Q-lattice structure in one spatial direction, we generalize it to a two dimensional lattice model which in general can be anisotropic in $x-y$ plane. And then, the Dirac equations over this Q-lattice background are deduced in section 3. In section 4, we present our numerical results for the fermionic spectral function. We conclude in section 5 with a summary of main results and suggestions for future research.

\section{Holographic Q-lattice geometry}

Here we are interested in a holographic Q-lattice geometry with no translational symmetry in both of the spatial directions. For this purpose, we consider a system containing two complex scalar fields $\phi_{1}$ and $\phi_{2}$ plus a Maxwell field $A_{\mu}$. A similar construction can also be seen in [32]. The simplest action may take the form as,

$$
S=\int d^{4} x \sqrt{-g}\left[R+6-\frac{1}{2} F^{2}-\left|\partial \phi_{1}\right|^{2}-m_{1}^{2}\left|\phi_{1}\right|^{2}-\left|\partial \phi_{2}\right|^{2}-m_{2}^{2}\left|\phi_{2}\right|^{2}\right],
$$

where $F=d A$. The equations of motion can be derived from the above action as follows

$$
\begin{aligned}
R_{\mu \nu} & =g_{\mu \nu}\left(-3+\frac{m_{1}^{2}}{2}\left|\phi_{1}\right|^{2}+\frac{m_{2}^{2}}{2}\left|\phi_{2}\right|^{2}\right)+\partial_{(\mu} \phi_{1} \partial_{\nu)} \phi_{1}^{*}+\partial_{(\mu} \phi_{2} \partial_{\nu)} \phi_{2}^{*}+\left(F_{\mu}{ }^{\rho} F_{\nu \rho}-\frac{1}{4} g_{\mu \nu} F^{2}\right), \\
\nabla_{\mu} F^{\mu \nu} & =0, \quad\left(\nabla^{2}-m_{1}^{2}\right) \phi_{1}=0, \quad\left(\nabla^{2}-m_{2}^{2}\right) \phi_{2}=0 .
\end{aligned}
$$

Consider the following ansatz

$$
\begin{gathered}
d s^{2}=-g_{t t}(z) d t^{2}+g_{z z}(z) d z^{2}+g_{x x}(z) d x^{2}+g_{y y}(z) d y^{2}, \\
A=A_{t}(z) d t, \quad \phi_{1}=e^{i k_{1} x} \varphi_{1}, \quad \phi_{2}=e^{i k_{2} y} \varphi_{2},
\end{gathered}
$$

\footnotetext{
${ }^{1}$ MIT is also observed in helical lattice model [42] and holographic charge density waves [43]. In addition, a simpler construction which breaks the translational invariance can be found in [44, 45], in which the lattice amplitude is absent, and thus no MIT happens in these models.
} 
with

$$
\begin{aligned}
& g_{t t}(z)=\frac{(1-z) P(z) Q(z)}{z^{2}}, \quad g_{z z}(z)=\frac{1}{z^{2}(1-z) P(z) Q(z)}, \\
& g_{x x}(z)=\frac{V_{1}(z)}{z^{2}}, \quad g_{y y}(z)=\frac{V_{2}(z)}{z^{2}}, \quad A_{t}(z)=\mu(1-z) a(z), \\
& P(z)=1+z+z^{2}-\frac{\mu^{2}}{2} z^{3},
\end{aligned}
$$

and substitute them into (2.2), one has five second order ODEs for $V_{1}, V_{2}, a, \varphi_{1}, \varphi_{2}$ and one first order ODE for $Q$. Note that in above ansatz, $k_{1}$ and $k_{2}$ are two wavenumbers along $x$ and $y$ directions, respectively, such that $\phi_{1}$ is periodic in $x$ direction with a lattice constant $2 \pi / k_{1}$ and $\phi_{2}$ is periodic in $y$ direction with a lattice constant $2 \pi / k_{2}$. In addition, in our holographic setup, the dual CFT involves two complex scalar operators with scaling dimension $\Delta_{1,2}=3 / 2 \pm\left(9 / 4+m_{1,2}^{2}\right)^{1 / 2}$. When either of the masses falls in the interval $[-9 / 4,-3 / 2)$, the $A d S_{2} \mathrm{BF}$ bound near the horizon will be violated by the scalar field, possibly leading to a different ground state. To avoid this possibility, we will set $m_{1,2}^{2}=-5 / 4$ for definiteness in our paper, which corresponds to the scaling dimension of $\Delta_{1,2+}=5 / 2$ and $\Delta_{1,2-}=1 / 2$.

To solve the ODEs numerically, we impose a regular boundary condition at the horizon $z=1$ and impose the following conditions on the conformal boundary

$$
Q(0)=1, \quad V_{1}(0)=V_{2}(0)=1, \quad a(0)=\mu .
$$

In this paper, we only focus on the standard quantisation of the scalar field, in which the asymptotic behaviors of $\varphi_{1}$ and $\varphi_{2}$ look like $\varphi_{1}=\lambda_{1} z^{\Delta_{1-}}$ and $\varphi_{2}=\lambda_{2} z^{\Delta_{2-}}$, respectively. The UV behavior of the scalar field corresponds to a Q-lattice deformation with lattice amplitudes $\lambda_{1,2}$. In addition, the temperature of the black hole is given by

$$
T=\frac{P(1) Q(1)}{4 \pi} .
$$

As a result, each of our Q-lattice solutions is specified by five dimensionless quantities $T / \mu$, $\lambda_{1} / \mu^{\Delta_{1-}}, \lambda_{2} / \mu^{\Delta_{2-}}, k_{1} / \mu$ and $k_{2} / \mu$. For simpleness, we shall still use $T, \lambda_{1,2}$ and $k_{1,2}$ to denote the above five dimensionless quantities in what follows.

\section{Dirac equation}

To explore the properties of fermionic spectral function in the Q-lattice geometry, we consider the following action including the dipole coupling with strength $p$ between the fermion and gauge field [20, 21, 46, 47]

$$
S_{D}=i \int d^{4} x \sqrt{-g} \bar{\zeta}\left(\Gamma^{a} \mathcal{D}_{a}-m_{\zeta}-i p \not F\right) \zeta
$$

where $\mathcal{D}_{a}=\partial_{a}+\frac{1}{4}\left(\omega_{\mu \nu}\right)_{a} \Gamma^{\mu \nu}-i q A_{a}$ and $\not{F}=\frac{1}{2} \Gamma^{\mu \nu}\left(e_{\mu}\right)^{a}\left(e_{\nu}\right)^{b} F_{a b}$ with $\left(e_{\mu}\right)^{a}$ and $\left(\omega_{\mu \nu}\right)_{a}$ being a set of orthogonal normal vector bases and the spin connection 1-forms, respectively. Here, 
the fermion is a probe field. Note that we have set the gauge coupling constant $g_{F}=\sqrt{2}$ here. It is different from the conventions in $[20,21]$, in which they set $g_{F}=1$. Consequently the charge $q$ and dipole coupling $p$ here will correspond to $q / \sqrt{2}$ and $p / \sqrt{2}$ in $[20,21]$ as the relevant quantities are the products $g_{F} q$ and $g_{F} p$.

The Dirac equation can be deduced from the above action

$$
\Gamma^{a} \mathcal{D}_{a} \zeta-m_{\zeta} \zeta-i p \not F \zeta=0 .
$$

To cancel off the spin connection, we can make a redefinition of $\zeta=\left(g_{t t} g_{x x} g_{y y}\right)^{-\frac{1}{4}} \mathcal{F}$. At the same time, by the Fourier expansion,

$$
\mathcal{F}=\int \frac{d \omega d k_{x} d k_{y}}{2 \pi} F(z, \mathbf{k}) e^{-i \omega t+i k_{x} x+i k_{y} y},
$$

where $\mathbf{k}=\left(-\omega, k_{x}, k_{y}\right)$, one has

$$
\begin{aligned}
-\frac{1}{\sqrt{g_{z z}}} \Gamma^{3} \partial_{z} F(z, \mathbf{k})+\frac{1}{\sqrt{g_{t t}}} \Gamma^{0}\left(-i \omega-i q A_{t}\right) F(z, \mathbf{k})+\frac{1}{\sqrt{g_{x x}}} \Gamma^{1} i k_{x} F(z, \mathbf{k}) \\
\quad+\frac{1}{\sqrt{g_{y y}}} \Gamma^{2} i k_{y} F(z, \mathbf{k})-m_{\zeta} F(z, \mathbf{k})+\frac{i p}{\sqrt{g_{z z} g_{t t}}} \Gamma^{3} \Gamma^{0} \partial_{z} A_{t} F(z, \mathbf{k})=0 .
\end{aligned}
$$

Choose the following gamma matrices

$$
\begin{array}{lll}
\Gamma^{3}=\left(\begin{array}{cc}
-\sigma^{3} & 0 \\
0 & -\sigma^{3}
\end{array}\right), & \Gamma^{0}=\left(\begin{array}{cc}
i \sigma^{1} & 0 \\
0 & i \sigma^{1}
\end{array}\right), \\
\Gamma^{1}=\left(\begin{array}{cc}
-\sigma^{2} & 0 \\
0 & \sigma^{2}
\end{array}\right), & \Gamma^{2}=\left(\begin{array}{cc}
0 & \sigma^{2} \\
\sigma^{2} & 0
\end{array}\right),
\end{array}
$$

and split the 4-component spinor into two 2-component spinors as $F=\left(F_{1}, F_{2}\right)^{T}$, one has

$$
\begin{gathered}
\frac{1}{\sqrt{g_{z z}}} \partial_{z}\left(\begin{array}{l}
F_{1}(\mathbf{k}) \\
F_{2}(\mathbf{k})
\end{array}\right)-m_{\zeta} \sigma^{3} \otimes\left(\begin{array}{l}
F_{1}(\mathbf{k}) \\
F_{2}(\mathbf{k})
\end{array}\right)+\left(\omega+q A_{t}\right) \frac{1}{\sqrt{g_{t t}}} i \sigma^{2} \otimes\left(\begin{array}{l}
F_{1}(\mathbf{k}) \\
F_{2}(\mathbf{k})
\end{array}\right) \\
\mp k_{x} \frac{1}{\sqrt{g_{x x}}} \sigma^{1} \otimes\left(\begin{array}{l}
F_{1}(\mathbf{k}) \\
F_{2}(\mathbf{k})
\end{array}\right)+\frac{k_{y}}{\sqrt{g_{y y}}} \sigma^{1} \otimes\left(\begin{array}{l}
F_{2}(\mathbf{k}) \\
F_{1}(\mathbf{k})
\end{array}\right)+\frac{p}{\sqrt{g_{z z} g_{t t}}}\left(\partial_{z} A_{t}\right) \sigma^{1} \otimes\left(\begin{array}{l}
F_{1}(\mathbf{k}) \\
F_{2}(\mathbf{k})
\end{array}\right)=0 .
\end{gathered}
$$

Furthermore, by the decomposition $F_{\alpha} \equiv\left(\mathcal{A}_{\alpha}, \mathcal{B}_{\alpha}\right)^{T}$ with $\alpha=1$, 2, the above Dirac equation can be expressed as

$$
\begin{aligned}
&\left(\frac{1}{\sqrt{g_{z z}}} \partial_{z} \mp m_{\zeta}\right)\left(\begin{array}{l}
\mathcal{A}_{1} \\
\mathcal{B}_{1}
\end{array}\right) \pm\left(\omega+q A_{t}\right) \frac{1}{\sqrt{g_{t t}}}\left(\begin{array}{c}
\mathcal{B}_{1} \\
\mathcal{A}_{1}
\end{array}\right)+\frac{p}{\sqrt{g_{z z} g_{t t}}}\left(\partial_{z} A_{t}\right)\left(\begin{array}{c}
\mathcal{B}_{1} \\
\mathcal{A}_{1}
\end{array}\right) \\
&-\frac{k_{x}}{\sqrt{g_{x x}}}\left(\begin{array}{c}
\mathcal{B}_{1} \\
\mathcal{A}_{1}
\end{array}\right)+\frac{k_{y}}{\sqrt{g_{y y}}}\left(\begin{array}{c}
\mathcal{B}_{2} \\
\mathcal{A}_{2}
\end{array}\right)=0 \\
&\left(\frac{1}{\sqrt{g_{z z}}} \partial_{z} \mp m_{\zeta}\right)\left(\begin{array}{c}
\mathcal{A}_{2} \\
\mathcal{B}_{1}
\end{array}\right) \pm\left(\omega+q A_{t}\right) \frac{1}{\sqrt{g_{t t}}}\left(\begin{array}{c}
\mathcal{B}_{2} \\
\mathcal{A}_{2}
\end{array}\right)+\frac{p}{\sqrt{g_{z z} g_{t t}}}\left(\partial_{z} A_{t}\right)\left(\begin{array}{c}
\mathcal{B}_{2} \\
\mathcal{A}_{2}
\end{array}\right) \\
&+\frac{k_{x}}{\sqrt{g_{x x}}}\left(\begin{array}{c}
\mathcal{B}_{2} \\
\mathcal{A}_{2}
\end{array}\right)+\frac{k_{y}}{\sqrt{g_{y y}}}\left(\begin{array}{c}
\mathcal{B}_{1} \\
\mathcal{A}_{1}
\end{array}\right)=0
\end{aligned}
$$


At the horizon, we can find that

$$
\partial_{z}\left(\begin{array}{c}
\mathcal{A}_{\alpha}(z, \mathbf{k}) \\
\mathcal{B}_{\alpha}(z, \mathbf{k})
\end{array}\right) \pm \frac{\omega}{4 \pi T} \frac{1}{1-z}\left(\begin{array}{c}
\mathcal{B}_{\alpha}(z, \mathbf{k}) \\
\mathcal{A}_{\alpha}(z, \mathbf{k})
\end{array}\right)=0 .
$$

In order to obtain the retarded Green function on the boundary by holography, the independent ingoing boundary condition should be imposed at the horizon, i.e.,

$$
\left(\begin{array}{c}
\mathcal{A}_{\alpha}(z, \mathbf{k}) \\
\mathcal{B}_{\alpha}(z, \mathbf{k})
\end{array}\right)=c_{\alpha}\left(\begin{array}{c}
1 \\
-i
\end{array}\right)(1-z)^{-\frac{i \omega}{4 \pi T}}
$$

Near the AdS boundary, the Dirac field reduces to

$$
\left(\begin{array}{l}
\mathcal{A}_{\alpha} \\
\mathcal{B}_{\alpha}
\end{array}\right) \approx a_{\alpha} z^{m_{\zeta}}\left(\begin{array}{l}
1 \\
0
\end{array}\right)+b_{\alpha} z^{-m_{\zeta}}\left(\begin{array}{l}
0 \\
1
\end{array}\right) .
$$

And so by holography, the retarded Green function can be read off

$$
a_{\alpha}=G_{\alpha \alpha^{\prime}} b_{\alpha^{\prime}}
$$

Note that since the four components of the Dirac fields couple to one another, we need to construct a basis of finite solutions, $\left(\mathcal{A}_{\alpha}^{I}, \mathcal{B}_{\alpha}^{I}\right)$ and $\left(\mathcal{A}_{\alpha}^{I I}, \mathcal{B}_{\alpha}^{I I}\right)$, to obtain the boundary Green function. We are mainly interested in the measurable spectral function, which is $A\left(\omega, k_{x}, k_{y}\right) \sim \operatorname{Im}(\operatorname{Tr} G)$.

\section{Mott transition on Q-lattice}

In this section, we study the properties of spectral function on Q-lattice. We shall firstly address Mott transition in an isotropic Q-lattice, in which we set $\lambda_{1}=\lambda_{2}$ and $k_{1}=k_{2}$. So, in this paper, we shall denote $\lambda=\lambda_{1}=\lambda_{2}$ and $k=k_{1}=k_{2}$ except in subsection 4.4, where we give a brief discussion on anisotropic spectral function. For definiteness, we work exclusively with the massless fermion and fix $q=1$. In addition, we work at a very low but non-zero temperature of $T \simeq 0.00398$ for this paper except the subsection 4.3 , in which we explore the dynamics at different temperatures.

\subsection{Free fermionic spectral function}

In this subsection, we present the results for free fermionic spectral function on Q-lattice. Because the notion of Fermi surface is only well defined at zero temperature, for our Qlattice system of low but non-zero temperature, we need an operational definition, which has been proposed in [48] and adopted in holographic models [40, 49]. It is argued that the Fermi momentum $k_{F}$ can be determined by searching the peak of $A(\omega, k)$ with a tiny frequency $\omega$. With the use of this operational definition, we show the $3 \mathrm{D}$ plot of spectral function $A\left(k_{x}, k_{y}\right)$ for a tiny $\omega$ in the left plot in figure 1 . We can see that the shape of Fermi surface is a circle, which is in agreement with the fact that our holographic Q-lattice geometry is isotropic. For convenience, we shall set $k_{y}=0$ in what follows. As an example, 

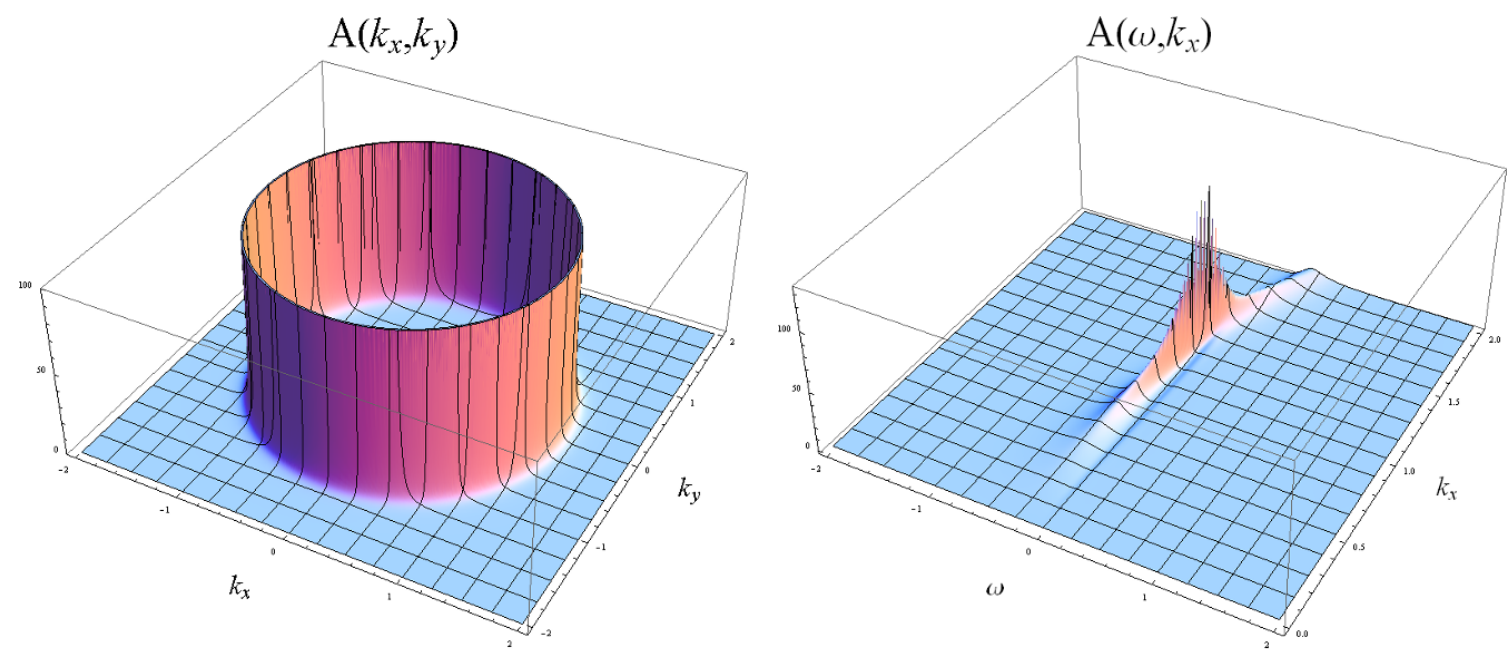

Figure 1. Left plot: the spectral function $A\left(k_{x}, k_{y}\right)$ for a tiny $\omega$. Right plot: the spectral function $A\left(\omega, k_{x}\right)$ for $k_{y}=0$. Here, we have fixed $\lambda=0.5$ and $k=0.8$.

the 3D plot of $A\left(\omega, k_{x}\right)$ is shown in the right plot in figure 1. A peak occurs near $\omega=0$ and $k_{F} \simeq 1.359$, indicating a Fermi surface.

The Q-lattice effects on the evolution of the free fermionic spectral function can be seen as follows. On one hand, one can fix the wave-number and see the changes of spectrum with the lattice amplitude. We show an example on the left plot in figure 2 , where $k=0.8$. For large $\lambda(\lambda=2$, blue line in the left plot in figure 2), only a small bump is displayed. With the decrease of $\lambda$, the small bump grows into a peak, which indicates the emergence of the Fermi surface. On the other hand, one can fix the lattice amplitude and adjust the wave-number $k$ to see its impact on the shape and location of Fermi surface. From the right plot in figure 2, we see that with the augmentation of the wave-number $k$, the Fermi peak shifts to positions with larger momenta. Moreover, we notice that the height of peak for $k=0.8$ is lower than that for $k=0.1$ or $k=1$. It appears to be a turning point around $k=0.8$. In the subsequent subsection, such a turning point can be further confirmed by studying the critical value $p_{c}$ of the onset of the gap and the gap width $\Delta / \mu$.

Finally, we would like to point out that no matter how to tune the lattice amplitude $\lambda$ and wave-number $k$, one can not observe a gap open at the Fermi level $(\omega \simeq 0)$, even though the Q-lattice background is dual to a deep insulating phase. It implies that in the absence of coupling, the holographic Q-lattice background itself is not able to drive fermionic probes to undergo a Mott transition. To model Mott physics, we shall introduce the dipole coupling term as proposed in [20].

\subsection{The Mott transition}

A Mott insulator is signaled by a vanishing spectral weight, i.e., a suppression of $A(\omega)$ around $\omega=0$. The 3D plot of spectral function $A\left(\omega, k_{x}\right)$ with $p=4.5$ for $\lambda=0.5$ and $k=0.8$ in figure 3 shows such a gap, which has previously been observed in RN-AdS geometry $[20,21]$ and other backgrounds [22-27]. In addition, two bands are located in the 

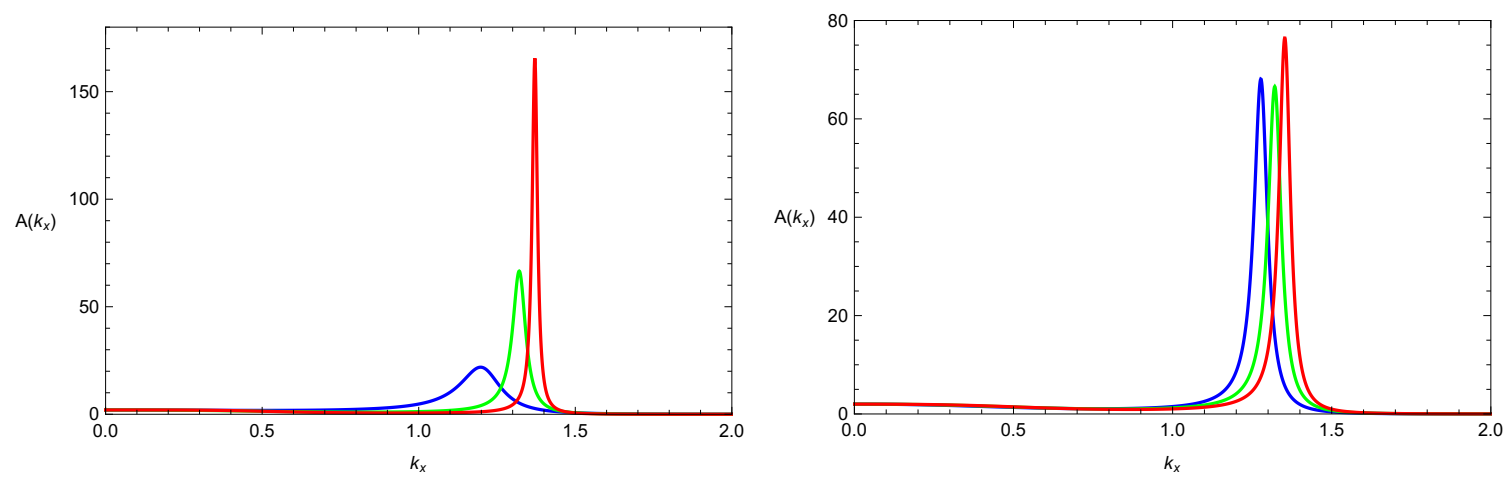

Figure 2. Left plot: the spectral function $A\left(k_{x}\right)$ for fixed $k=0.8$ and different $\lambda$ (red line for $\lambda=0.1$, green for $\lambda=1$ and blue for $\lambda=2)$. Right plot: the spectral function $A\left(k_{x}\right)$ for fixed $\lambda=1$ and different $k$ (red line for $k=1$, green for $k=0.8$ and blue for $k=0.1$ ). Here, all plots are for $k_{y}=0$ and a tiny $\omega$.

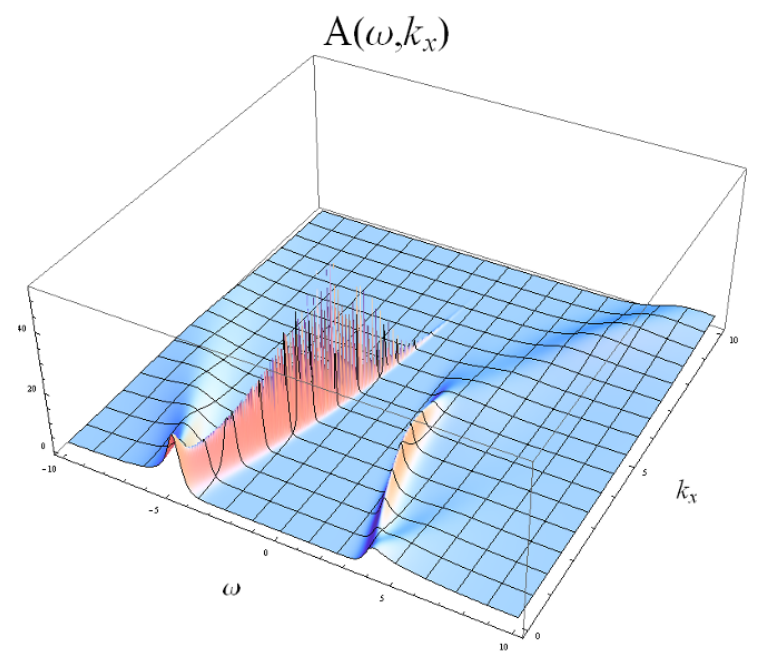

Figure 3. The spectral function $A\left(\omega, k_{x}\right)$ for $p=4.5$. Here, $\lambda=0.5, k=0.8$ and $k_{y}=0$. A gap is clearly visible around $\omega=0$.

positive frequency and negative frequency regions, respectively. Furthermore, we also show the spectral function $A\left(\omega, k_{x}\right)$ with fixed $p$ for sample values of $k_{x}$ in figure 4 . For $p=0$ (the left plot in figure 4), as the peak approaches $\omega=0$, it becomes sharper and narrower, indicating a Fermi surface at $k=k_{F}$. While the right plot in figure 4 shows the spectral function $A\left(\omega, k_{x}\right)$ with $p=4.5$ for sample values of $k_{x}$. It indicates that when $p$ exceeds certain critical point $p_{c}$, a gap opens and exists for all $k_{x}$. The above observation strongly manifests that a transition occurs from a (non)-Fermi like phase to an insulating phase.

To gain a better understanding of the spectral measure with dipole coupling on Qlattice, we study the density of states (DOS) for our fermionic system. The DOS $A(\omega)$ signals the total weight of the spectral measure and is defined by the integral of $A\left(\omega, k_{x}, k_{y}\right)$ over $k_{x}$ and $k_{y}$. Since we have set $k_{y}=0$, the DOS only probes the effects in $k_{x}$ direction. Figure 5 shows the DOS $A(\omega)$ with $\lambda=0.5$ and $k=0.8$, and exhibits the dynamical 

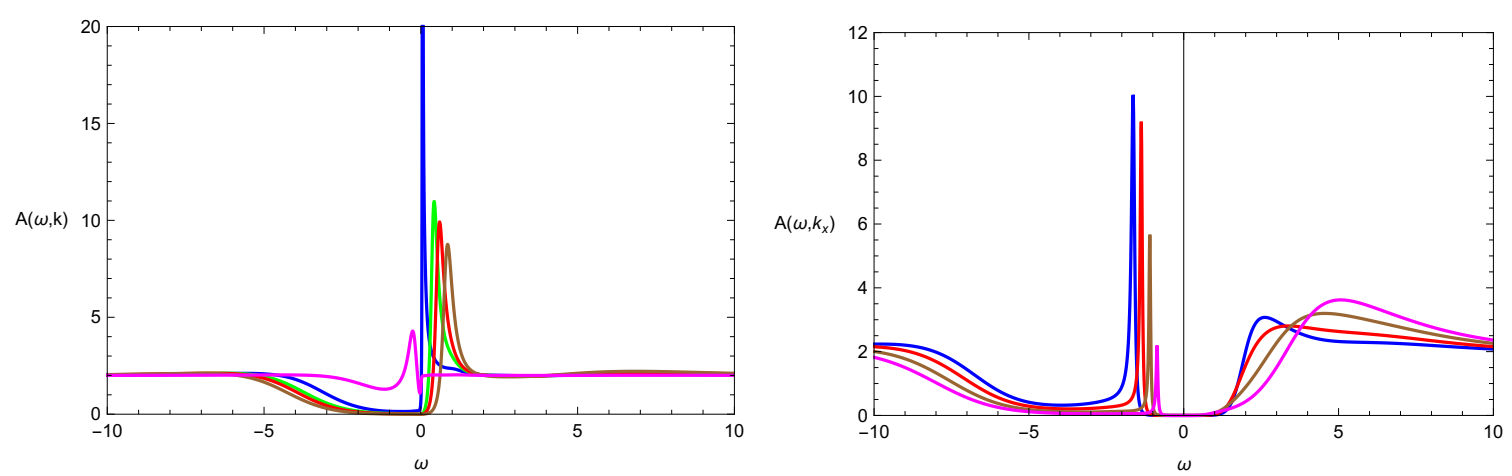

Figure 4. The spectral function $A\left(\omega, k_{x}\right)$ for sample values of $k_{x}$. Left plot is for $p=0$ and right for $p=4.5$. Here, $\lambda=0.5, k=0.8$ and $k_{y}=0$.

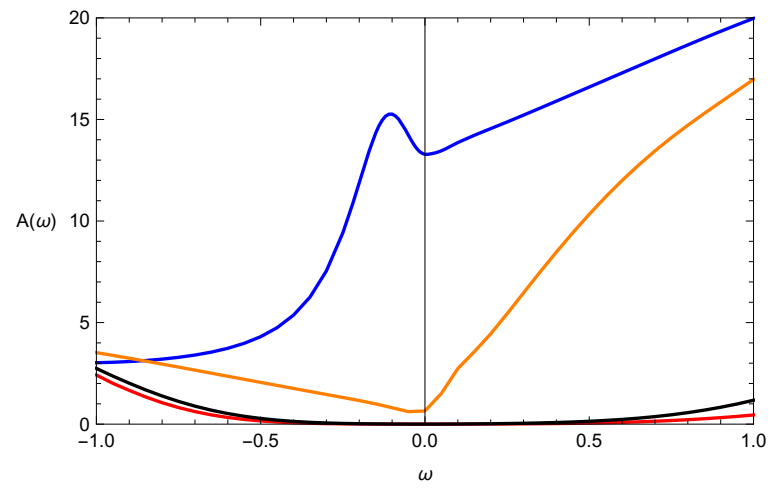

Figure 5. The DOS $A(\omega)$ with $\lambda=0.5$ and $k=0.8$ for $p=0$ (blue), $p=1.5$ (orange), $p=4$ (black) and $p=4.5$ (red).

generation of a gap with the increase of the diploe coupling $p$. More importantly, as $p$ increases, the spectral weight switches gradually from the positive frequency region to the negative frequency region. The dynamical generation and spectral weight transfer are two key features of doped Mott physics. Such observations are confirmed by checking other values of lattice parameters $\lambda$ and $k$. Therefore, the Mott transition in holographic Qlattice fermionic system can be implemented by introducing a dipole coupling, which is supposed to play double roles of doping as well as on-site interaction like $U$ in Hubbard model, as argued in RN-AdS geometry [20, 21].

Now, we turn to study the critical value $p_{c}$ of Mott transition, which reflects how easy it is to open the gap dynamically. In numerical calculations, the onset of gap can be identified as that the DOS $A(\omega)$ at the Fermi level drops below some small number (here, we take $10^{-3}$ ). With this in mind, we find that the critical value $p_{c} \simeq 3.98$ for $\lambda=0.5$ and $k=0.8$, which corresponds to a metallic phase in [30]. While for an insulating phase with $\lambda=2$ and $k=1 / 2^{3 / 2}, p_{c}=3.74$. It implies that the gap opens easier in a deep insulating phase than metallic phase. This observation can be further confirmed by evaluating the critical value for various wave number $k$ and lattice amplitude $\lambda$, as we list in table 1 and 2 . Nevertheless, we notice that an anomalous behavior occurs for $k \geq k_{c}$ ( where $k_{c}$ is the 


\begin{tabular}{|c|c|c|c|c|c|c|}
\hline$k$ & 0.1 & 0.3 & 0.5 & 0.8 & 1 & 1.5 \\
\hline$p_{c}$ & 3.44 & 3.76 & 4.08 & 4.12 & 4.08 & 3.93 \\
\hline
\end{tabular}

Table 1. $p_{c}$ for sample values of $k$ and fixed $\lambda=1$.

\begin{tabular}{|c|c|c|c|c|c|c|}
\hline$\lambda$ & 0.1 & 0.5 & 1 & 1.5 & 2 & 3 \\
\hline$p_{c}$ & 3.90 & 3.83 & 3.76 & 3.7 & 3.64 & 3.55 \\
\hline
\end{tabular}

Table 2. $p_{c}$ for sample values of $\lambda$ and fixed $k=0.3$.

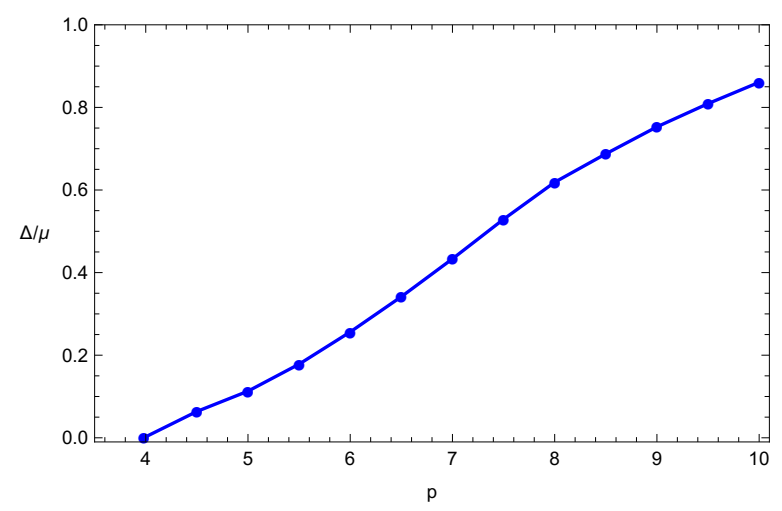

Figure 6. The width $\Delta / \mu$ of the gap as a function of the dipole coupling $p$ for $\lambda_{1}=\lambda_{2}=0.5$ and $k_{1}=k_{2}=0.8$.

critical value for MIT at zero temperature, with $k_{c} \simeq 0.8$ for $\lambda=1$ ), in which the critical value $p_{c}$ diminishes slightly with the increase of $k$, as opposite to that for $k<k_{c}$.

Subsequently, we shall study the features of Mott insulating phase over a Q-lattice background. A characteristic measure is the width of the gap $\Delta / \mu$. Figure 6 shows the width $\Delta / \mu$ as a function of the dipole coupling $p$ for $\lambda=0.5$ and $k=0.8$. As $p$ increases, the width of the gap becomes larger. It is just the effects of on-site interaction strength $U$ of Hubbard model, which $p$ plays.

We also study the effects on the width of the gap of wave-number $k$ and lattice amplitude $\lambda$, which can be summarized as follows.

- First of all, a turning point is observed in the plot of gap width $\Delta / \mu$ versus $k$, which is shown in figure 7. Specifically, as $k$ increases from small one, the width of the gap $\Delta / \mu$ will decrease at first, which coincides with the idea of Mott that the strength of electron-electron correlations increases with the decrease of lattice constant [15-19]. However, when the wave-number exceeds a critical value $k_{c}, \Delta / \mu$ rises up slightly. Interestingly enough, we find the values of $k_{c}$ falls into the critical regime of MIT in the phase diagram of the background, which is quite general. We expect to understand this anomaly with an analytical treatment in near future.

- The critical value $k_{c}$ is almost independent of the dipole coupling $p$ but becomes larger with the increase of the lattice amplitude, as shown in figure 7 and table 3 . 

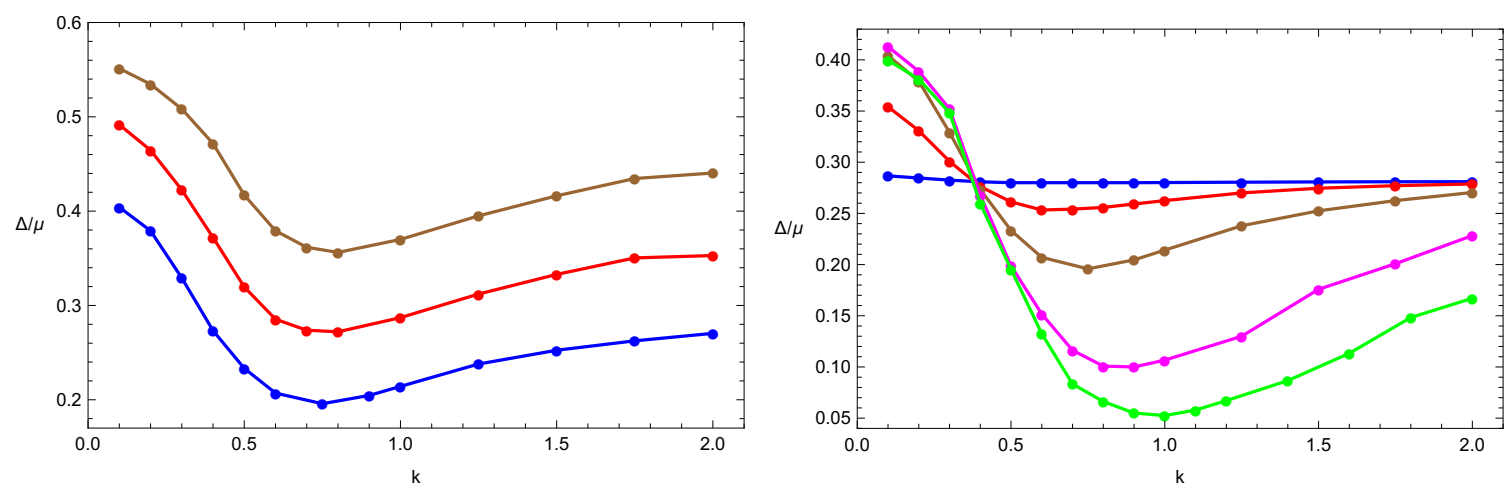

Figure 7. The width $\Delta / \mu$ of the gap as a function of $k$. Left plot is for $\lambda=1$ and different $p$ (blue line for $p=6$, red for $p=6.5$ and brown for $p=7$ ) and right plot for $p=6$ and different $\lambda$ (blue line for $\lambda=0.1$, red for $\lambda=0.5$, brown for $\lambda=1$, magnetic for $\lambda=2$ and green for $\lambda=3$ ).

\begin{tabular}{|c|c|c|c|c|c|c|}
\hline$\lambda$ & 0.1 & 0.5 & 0.8 & 1 & 2 & 3 \\
\hline$k_{c}$ & 0.5 & 0.6 & 0.7 & 0.8 & 0.9 & 1 \\
\hline
\end{tabular}

Table 3. The turning point $k_{c}$ for sample values of $\lambda$ and fixed $p=6$.

In addition, it is interesting to notice that in the right plot of figure 7 all the lines almost intersect at one point of $k \simeq 0.38$.

- The width of gap as a function of the lattice amplitude $\lambda$ is plotted in figure 8. For $k<0.38$, with the augmentation of the lattice amplitude $\lambda$, the width of the gap $\Delta / \mu$ rises up at first and then tends to a constant or very slightly decreases, depending on the dipole coupling $p$. But for $k>0.38$ the cases are just opposite.

- As a consistent check, one may find that in the right plot in figure 8 , all the lines converge to a point at $\lambda \rightarrow 0$, which just corresponds to the gap width in RN-AdS black hole background.

\subsection{The dynamics at different temperatures}

So far, we have only focused on the system at a very low temperature $T \simeq 0.00398$. Now, we turn to study the evolution of the spectral function with temperature. The temperature dynamics is one of important aspects of Mott insulators. A characteristic quantity is the ratio of the zero-temperature gap $\Delta$ to the critical temperature $T_{*}$, at which the gap closes. A typical example is transition-metal oxides $V O_{2}$, for which the ratio $\Delta / T_{*}$ is approximately 20. Compared with the case of superconductor, in which the U(1) symmetry is spontaneously broken and $\Delta / T_{c} \simeq 1-2$, the ratio $\Delta / T_{*}$ is well beyond unity, which is one of the unresolved puzzles with $\mathrm{VO}_{2}$. It indicates that the driven force to form the gap in Mott transition is strongly correlated. By holography, the authors in [21] show the ratio $\Delta / T_{*}$ in RN-AdS black hole is approximately 10 for $p=6$ (or $p=7$ ), which suggests holography can provide a good description on strong correlation induced transition. Here we shall address this issue on Q-lattice by holography. 

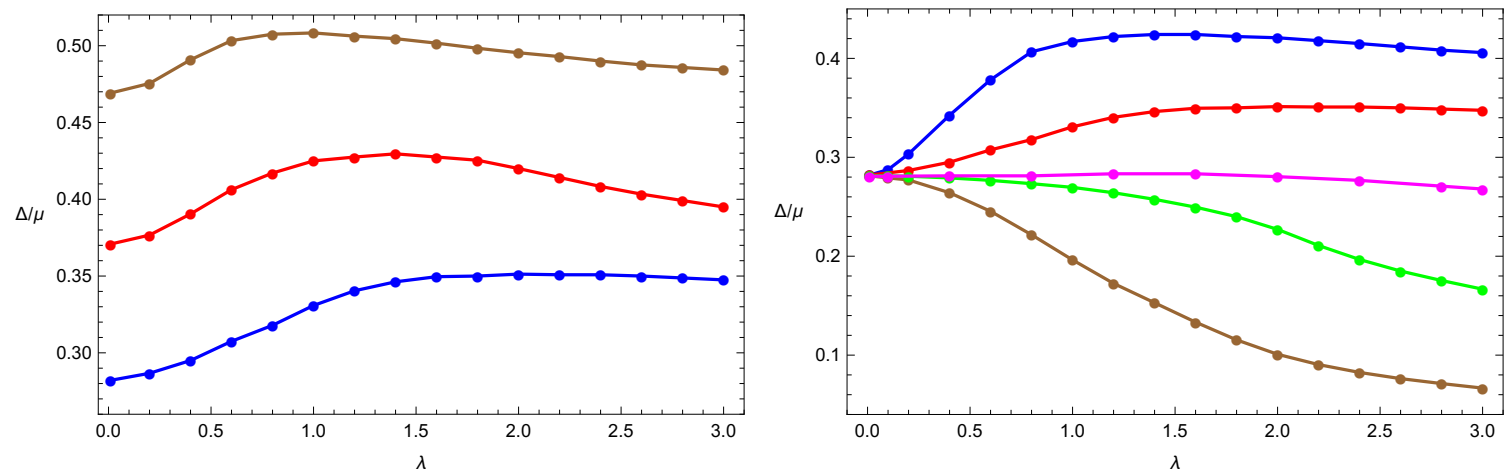

Figure 8. The width $\Delta / \mu$ of the gap as a function of $\lambda$. Left plot is for $k=0.3$ and different $p$ (blue line for $p=6$, red for $p=6.5$ and brown for $p=7$ ) and right plot is for $p=6$ and different $k$ (blue line for $k=0.03$, red for $k=0.3$, magenta for $k=0.38$, Green for $k=0.8$ and brown for $k=2)$.
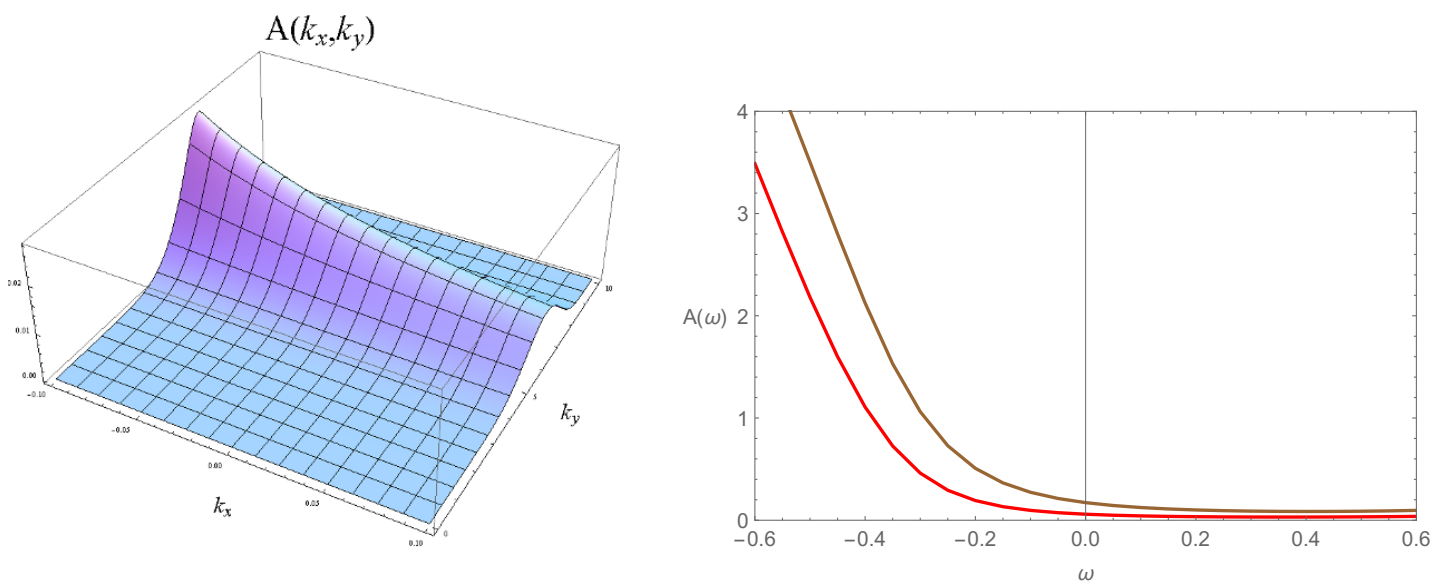

Figure 9. Left plot: the $3 \mathrm{D}$ plot for $T \simeq 0.115$. Right plot: the DOS for different $T$ (red for $T \simeq 0.115$ and brown for $T \simeq 0.151$ ). Here, $p=6, \lambda=1$ and $k=0.3$.

Before working out the results of the ratio $\Delta / T_{*}$, we first make a qualitative description. Figure 9 shows a $3 \mathrm{D}$ plot of the spectral function at $T \simeq 0.115$ and the DOS for different $T$ with $\lambda=1$ and $k=0.3$. It is obvious that the gap closes when the temperature exceeds some certain value. Now, we work out the ratio $\Delta / T_{*}$ for different lattice amplitudes $\lambda$ and wave-numbers $k$ for $p=6$ (table 4 ). ${ }^{2}$ From table 4 we can see that most values of the ratio $\Delta / T_{*}$ are approximately 10 . Especially, in the deep insulating background, the value of $\Delta / T_{*}$ is larger than others, approaching 11. In summary, in our holographic fermionic system with dipole coupling on Q-lattice, the temperature dynamics possesses non-trivial behavior, as revealed in RN-AdS black hole [21].

\footnotetext{
${ }^{2}$ Because we can not cool our system down to zero temperature, here we take the width of gap $\Delta$ at $T \simeq 0.00398$ instead of zero temperature gap.
} 


\begin{tabular}{|c|c|c|c|c|c|c|}
\hline$(\lambda, k)$ & $(1,0.1)$ & $(1,0.75)$ & $(1,2)$ & $(0.1,0.3)$ & $(1,0.3)$ & $(3,0.3)$ \\
\hline$\Delta / T_{*}$ & 10.93 & 8.86 & 10.24 & 9.79 & 10.33 & 10.07 \\
\hline
\end{tabular}

Table 4. The ratio $\Delta / T_{*}$ for different lattice amplitude $\lambda$ and wave-number $k$.
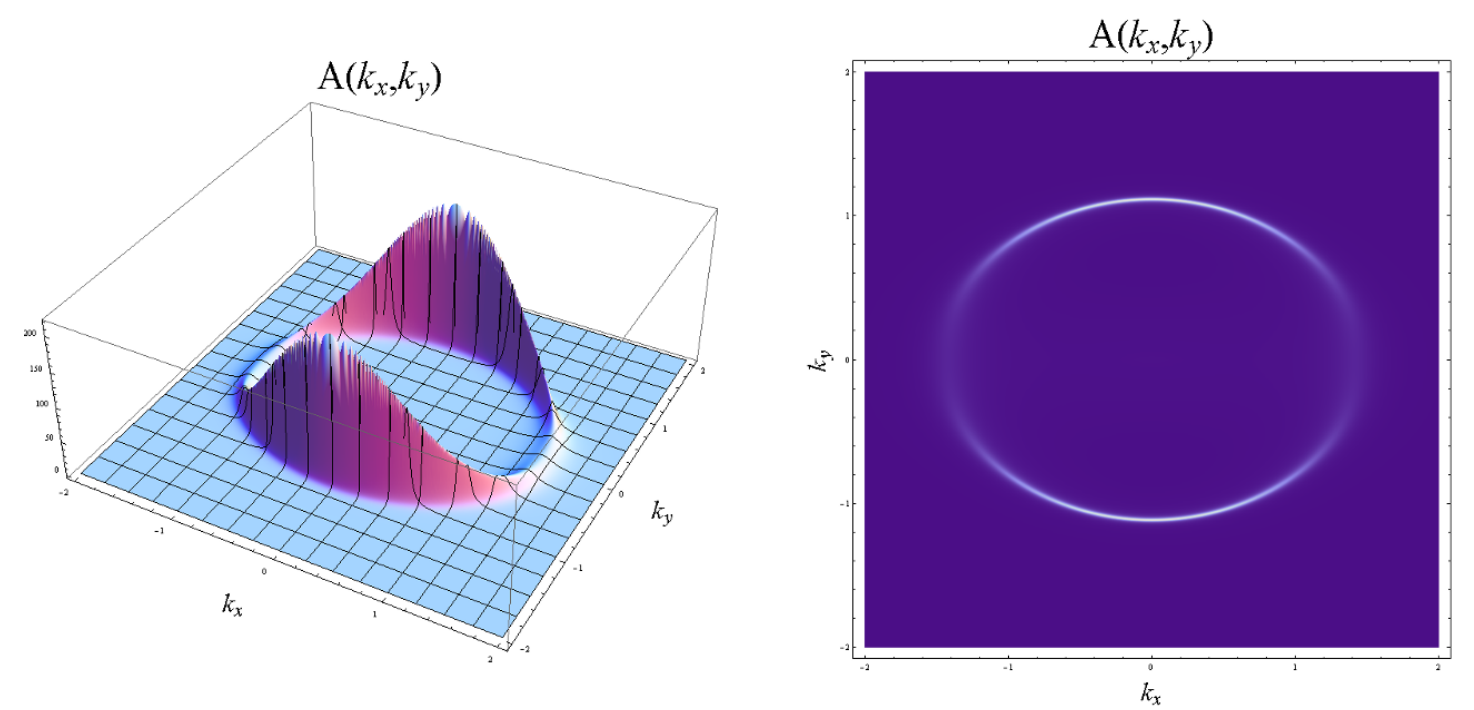

Figure 10. 3D and density plot of spectral function $A\left(k_{x}, k_{y}, \omega=0\right)$ on anisotropic Q-lattice for $p=0$. Here, $q=1, \lambda_{1}=2, \lambda_{2}=0.1$ and $k_{1}=k_{2}=0.8$.

\subsection{Fermi surface and gap on anisotropic Q-lattice}

In this subsection, we briefly discuss the Fermi surface and gap on anisotropic Q-lattice. As shown in figure 10, if we set $q=1, \lambda_{1}=2, \lambda_{2}=0.1$ and $k_{1}=k_{2}=0.8$ for $p=0$, we find that the Fermi peaks along $k_{x}$ direction develop into some bumps. It exhibits an anisotropic peaks with different magnitudes, which suggests that insulating phase arises in one direction while metallic in the other. Furthermore, when we switch on the dipole coupling $p$, the gap along $k_{x}$ direction gradually opens but the bump still remain along $k_{y}$ direction (figure 11). Finally, we would like to comment that the same statement is true for the conductivity on Q-lattice. For instance, since the lattice is introduced in just one direction, the Q-lattice geometries are highly anisotropic in [30], in which the calculation of the conductivity reveals that they can be insulators in the direction where the lattice is placed whilst remaining a pure metal in the other direction where the lattice is absence and the translational invariance is reserved. Therefore, the anisotropic geometry with insulating phase in one direction but metallic in the other provides us more space to model real materials with anisotropy by holography.

\section{Conclusion and discussion}

Our main results in this paper are:

- Two key features of doped Mott physics, the dynamical emergence of a gap and the spectral weight transfer, are observed in the Q-lattice background with dipole 

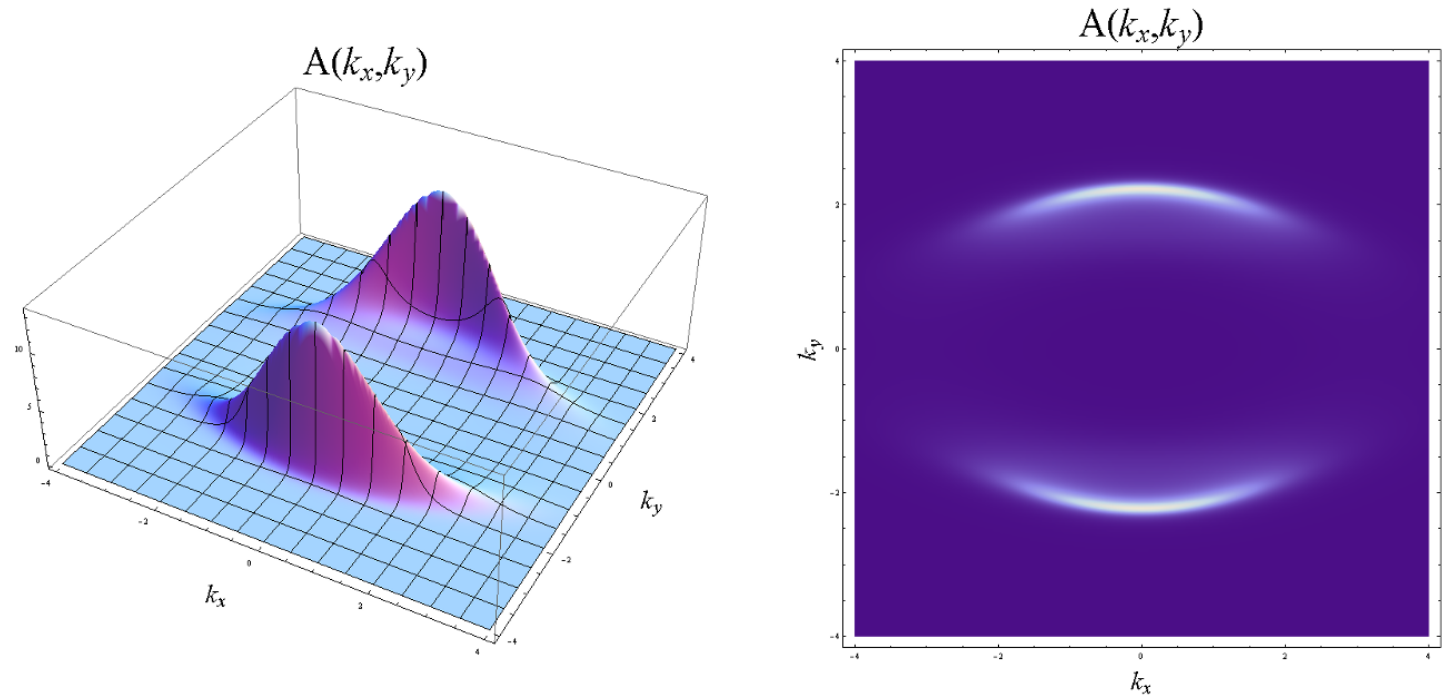

Figure 11. 3D and density plot of spectral function $A\left(k_{x}, k_{y}, \omega=0\right)$ on anisotropic Q-lattice for $p=1$. Here, $q=1, \lambda_{1}=2, \lambda_{2}=0.1$ and $k_{1}=k_{2}=0.8$.

coupling. Such features have been observed in many of holographic fermionic systems including dipole coupling, e.g., RN-AdS geometry and other geometry. It confirms the robustness of the generations of Mott gap induced by dipole coupling, which play the double roles of doping as well as the interaction strength $U$ in the Hubbard model.

- The most important thing is that the fermionic system with dipole coupling on Qlattice can exhibit abundant Mott physics due to the introduction of wave-number $k$ and lattice amplitude $\lambda$.

- The evolution of the spectral function as a function of temperature reveals the nontrivial temperature dynamical behavior, which indicates the Mott transition induced by dipole coupling on Q-lattice is due to strong correlations but not the spontaneous breaking of some symmetry.

- For the free fermionic system, the wave-number $k$ and the lattice amplitude $\lambda$ can not generate the Mott gap. When dipole coupling $p$ exceeds some critical value, the gap opens and, usually, the gap opens much easily in deep insulating phase.

- The anisotropic peaks with different magnitudes occurs in our holographic fermionic system on Q-lattice. It indicates that insulating and metallic phases arise in different directions, respectively.

Still, a number of problems deserve further exploration.

- Rather than the dipole coupling, we may introduce other sorts of couplings to induce the Mott transition. Especially the interaction of electron-phonon as $\bar{\zeta}\left(\eta_{1} \phi_{1}+\eta_{2} \phi_{2}\right) \zeta+$ h.c may have a manifest periodic structure and generate Brillouin zones. Our work on this subject is under progress. 
- By the poles and zeros duality through the $\operatorname{det} G_{R}$, the pseudo-gap phase can be observed in the holographic fermions with dipole coupling in RN-AdS geometry [50]. It is valuable to address this problem in the holographic Q-lattice model.

- It is interesting to study the Fermi arc phenomenology in the anisotropic Q-lattice model.

\section{Acknowledgments}

We are grateful to the anonymous referee for valuable comments. This work is supported by the Natural Science Foundation of China under Grant Nos.11275208, 11305018 and 11178002. Y.L. also acknowledges the support from Jiangxi young scientists (JingGang Star) program and 555 talent project of Jiangxi Province. J.P. Wu is also supported by Program for Liaoning Excellent Talents in University (No. LJQ2014123).

Open Access. This article is distributed under the terms of the Creative Commons Attribution License (CC-BY 4.0), which permits any use, distribution and reproduction in any medium, provided the original author(s) and source are credited.

\section{References}

[1] S.S. Gubser, Breaking an Abelian gauge symmetry near a black hole horizon, Phys. Rev. D 78 (2008) 065034 [arXiv:0801.2977] [INSPIRE].

[2] S.A. Hartnoll, C.P. Herzog and G.T. Horowitz, Building a Holographic Superconductor, Phys. Rev. Lett. 101 (2008) 031601 [arXiv:0803.3295] [INSPIRE].

[3] S.A. Hartnoll, C.P. Herzog and G.T. Horowitz, Holographic Superconductors, JHEP 12 (2008) 015 [arXiv:0810.1563] [INSPIRE].

[4] S.-S. Lee, A Non-Fermi Liquid from a Charged Black Hole: A Critical Fermi Ball, Phys. Rev. D 79 (2009) 086006 [arXiv: 0809.3402] [INSPIRE].

[5] H. Liu, J. McGreevy and D. Vegh, Non-Fermi liquids from holography, Phys. Rev. D 83 (2011) 065029 [arXiv:0903.2477] [inSPIRE].

[6] M. Cubrovic, J. Zaanen and K. Schalm, String Theory, Quantum Phase Transitions and the Emergent Fermi-Liquid, Science $\mathbf{3 2 5}$ (2009) 439 [arXiv: 0904.1993] [INSPIRE].

[7] J.P. Wu, Holographic fermions in charged Gauss-Bonnet black hole, JHEP 07 (2011) 106 [arXiv: 1103.3982] [INSPIRE].

[8] J.-P. Wu, Some properties of the holographic fermions in an extremal charged dilatonic black hole, Phys. Rev. D 84 (2011) 064008 [arXiv:1108.6134] [INSPIRE].

[9] W.-J. Li and J.-P. Wu, Holographic fermions in charged dilaton black branes, Nucl. Phys. B 867 (2013) 810 [arXiv:1203.0674] [INSPIRE].

[10] X.-M. Kuang, E. Papantonopoulos, B. Wang and J.-P. Wu, Formation of Fermi surfaces and the appearance of liquid phases in holographic theories with hyperscaling violation, JHEP 11 (2014) 086 [arXiv: 1409.2945] [INSPIRE].

[11] J.N. Laia and D. Tong, A Holographic Flat Band, JHEP 11 (2011) 125 [arXiv:1108.1381] [INSPIRE]. 
[12] W.-J. Li, R. Meyer and H.-b. Zhang, Holographic non-relativistic fermionic fixed point by the charged dilatonic black hole, JHEP 01 (2012) 153 [arXiv:1111.3783] [INSPIRE].

[13] M. Alishahiha, M.R. Mohammadi Mozaffar and A. Mollabashi, Fermions on Lifshitz Background, Phys. Rev. D 86 (2012) 026002 [arXiv:1201.1764] [INSPIRE].

[14] L.Q. Fang, X.-H. Ge, J.-P. Wu and H.-Q. Leng, Anisotropic Fermi surface from holography, arXiv:1409.6062 [INSPIRE].

[15] N.F. Mott and R. Peierls, Discussion of the paper by de Boer and Verwey, Proc. Phys. Soc. A 49 (1937) 72.

[16] N.F. Mott, The Basis of the Electron Theory of Metals, with Special Reference to the Transition Metals, Proc. Phys. Soc. A 62 (1949) 416.

[17] N.F. Mott, On the transition to metallic conduction in semiconductors, Can. J. Phys. 34 (1956) 1356.

[18] N.F. Mott, The transition to the metallic state, Philos. Mag. 6 (1961) 287.

[19] N.F. Mott, Metal-Insulator Transitions, Taylor and Francis, London/Philadelphia (1990).

[20] M. Edalati, R.G. Leigh and P.W. Phillips, Dynamically Generated Mott Gap from Holography, Phys. Rev. Lett. 106 (2011) 091602 [arXiv: 1010.3238] [INSPIRE].

[21] M. Edalati, R.G. Leigh, K.W. Lo and P.W. Phillips, Dynamical Gap and Cuprate-like Physics from Holography, Phys. Rev. D 83 (2011) 046012 [arXiv:1012.3751] [INSPIRE].

[22] J.-P. Wu and H.-B. Zeng, Dynamic gap from holographic fermions in charged dilaton black branes, JHEP 04 (2012) 068 [arXiv: 1201.2485] [INSPIRE].

[23] J.-P. Wu, Emergence of gap from holographic fermions on charged Lifshitz background, JHEP 04 (2013) 073 [INSPIRE].

[24] J.-P. Wu, The charged Lifshitz black brane geometry and the bulk dipole coupling, Phys. Lett. B 728 (2014) 450 [INSPIRE].

[25] X.-M. Kuang, B. Wang and J.-P. Wu, Dipole Coupling Effect of Holographic Fermion in the Background of Charged Gauss-Bonnet AdS Black Hole, JHEP 07 (2012) 125 [arXiv: 1205.6674] [INSPIRE].

[26] X.-M. Kuang, B. Wang and J.-P. Wu, Dynamical gap from holography in the charged dilaton black hole, Class. Quant. Grav. 30 (2013) 145011 [arXiv:1210.5735] [INSPIRE].

[27] L.Q. Fang, X.-H. Ge and X.-M. Kuang, Holographic fermions with running chemical potential and dipole coupling, Nucl. Phys. B 877 (2013) 807 [arXiv:1304.7431] [INSPIRE].

[28] W.-J. Li and H.-b. Zhang, Holographic non-relativistic fermionic fixed point and bulk dipole coupling, JHEP 11 (2011) 018 [arXiv:1110.4559] [INSPIRE].

[29] G. Vanacore and P.W. Phillips, Minding the Gap in Holographic Models of Interacting Fermions, Phys. Rev. D 90 (2014) 044022 [arXiv: 1405.1041] [INSPIRE].

[30] A. Donos and J.P. Gauntlett, Holographic Q-lattices, JHEP 04 (2014) 040 [arXiv: 1311.3292] [INSPIRE].

[31] S.R. Coleman, Q Balls, Nucl. Phys. B 262 (1985) 263 [Erratum ibid. B 269 (1986) 744] [INSPIRE].

[32] A. Donos and J.P. Gauntlett, Novel metals and insulators from holography, JHEP 06 (2014) 007 [arXiv: 1401.5077] [INSPIRE]. 
[33] M. Blake and A. Donos, Quantum Critical Transport and the Hall Angle, arXiv:1406.1659 [INSPIRE].

[34] A. Donos and J.P. Gauntlett, Thermoelectric DC conductivities from black hole horizons, JHEP 11 (2014) 081 [arXiv: 1406.4742] [INSPIRE].

[35] Y. Ling, P. Liu, C. Niu, J.-P. Wu and Z.-Y. Xian, Holographic Superconductor on Q-lattice, arXiv:1410.6761 [INSPIRE].

[36] G.T. Horowitz, J.E. Santos and D. Tong, Optical Conductivity with Holographic Lattices, JHEP 07 (2012) 168 [arXiv:1204.0519] [INSPIRE].

[37] G.T. Horowitz, J.E. Santos and D. Tong, Further Evidence for Lattice-Induced Scaling, JHEP 11 (2012) 102 [arXiv:1209.1098] [INSPIRE].

[38] G.T. Horowitz and J.E. Santos, General Relativity and the Cuprates, arXiv:1302.6586 [INSPIRE].

[39] Y. Ling, C. Niu, J.-P. Wu and Z.-Y. Xian, Holographic Lattice in Einstein-Maxwell-Dilaton Gravity, JHEP 11 (2013) 006 [arXiv:1309.4580] [INSPIRE].

[40] Y. Ling, C. Niu, J.-P. Wu, Z.-Y. Xian and H.-b. Zhang, Holographic Fermionic Liquid with Lattices, JHEP 07 (2013) 045 [arXiv: 1304.2128] [INSPIRE].

[41] A. Donos and J.P. Gauntlett, The thermoelectric properties of inhomogeneous holographic lattices, arXiv:1409.6875 [INSPIRE].

[42] A. Donos and S.A. Hartnoll, Interaction-driven localization in holography, Nature Phys. 9 (2013) 649 [arXiv:1212.2998] [INSPIRE].

[43] Y. Ling, C. Niu, J. Wu, Z. Xian and H.-b. Zhang, Metal-insulator Transition by Holographic Charge Density Waves, Phys. Rev. Lett. 113 (2014) 091602 [arXiv: 1404.0777] [INSPIRE].

[44] T. Andrade and B. Withers, A simple holographic model of momentum relaxation, JHEP 05 (2014) 101 [arXiv:1311.5157] [INSPIRE].

[45] B. Goutéraux, Charge transport in holography with momentum dissipation, JHEP 04 (2014) 181 [arXiv: 1401.5436] [INSPIRE].

[46] I. Bah, A. Faraggi, J.I. Jottar, R.G. Leigh and L.A. Pando Zayas, Fermions and D $=11$ Supergravity On Squashed Sasaki-Einstein Manifolds, JHEP 02 (2011) 068 [arXiv: 1008.1423] [INSPIRE].

[47] I. Bah, A. Faraggi, J.I. Jottar and R.G. Leigh, Fermions and Type IIB Supergravity On Squashed Sasaki-Einstein Manifolds, JHEP 01 (2011) 100 [arXiv:1009.1615] [INSPIRE].

[48] A. Kanigel et al., Evolution of the pseudogap from Fermi arcs to the nodal liquid, Nature Phys. 2 (2006) 447.

[49] F. Benini, C.P. Herzog and A. Yarom, Holographic Fermi arcs and a d-wave gap, Phys. Lett. B 701 (2011) 626 [arXiv:1006.0731] [InSPIRE].

[50] J. Alsup, E. Papantonopoulos, G. Siopsis and K. Yeter, Duality between zeroes and poles in holographic systems with massless fermions and a dipole coupling, arXiv:1404.4010 [INSPIRE]. 